\title{
The temporal impact of aging on the burden of herpes zoster
}

\author{
Lijoy Varghese ${ }^{1 *}$ (D, Baudouin Standaert ${ }^{2}$, Antonio Olivieri ${ }^{2,3}$ and Desmond Curran ${ }^{2}$
}

\begin{abstract}
Background: The risk of Herpes Zoster $(\mathrm{HZ})$ increases with age and various studies have also demonstrated an increasing $\mathrm{HZ}$ incidence globally. Simultaneously, the global trend of an aging population has placed a greater burden on the healthcare system. This study aims to estimate the potential burden of HZ over time accounting for the increasing trends of both $\mathrm{HZ}$ incidence and global aging.

Methods: A recent systematic review on $\mathrm{HZ}$ incidence identified studies that evaluated the temporal effects of $\mathrm{HZ}$ incidence. Data from the identified studies were extracted to estimate the trend of $\mathrm{HZ}$ incidence in the $\geq 65$-year-old age cohort. The incidence rates were estimated up to the year 2030 using two scenarios: a linear extrapolation and a last observation carried forward. Three countries were chosen to perform the analysis on: Australia, Japan and the United States.

Results: The incidence data from the three countries showed an average annual increase between 2.35 and 3.74\% over the time period of the studies selected. The elderly population for the US, Japan and Australia are expected to increase by 55, 10 and 53\% respectively by the year 2030 compared to the levels in 2015. Under the first scenario between 2001 and 2030, the number of annual incident cases of $\mathrm{HZ}$ in those aged $\geq 65$ years is expected to increase by $+343 \%(293,785$ to $1,303,328)$, $+176 \%(158,616$ to 437,867$)$ and $+376 \%(18,105$ to 86,268$)$ in the US, Japan and Australia respectively while those for the second scenario are $+150 \%,+83 \%$ and $+223 \%$ respectively. In the US alone, the estimated annual cost of HZ-related cases in the $\geq 65$ age cohort is approximately 4.74 Billion US\$ in 2030.
\end{abstract}

Conclusions: The increasing incidence of $\mathrm{HZ}$ coupled with the demographic trends (i.e., aging population and greater life expectancy) in many countries are likely to imply a rising economic burden of $\mathrm{HZ}$ on already constrained healthcare budgets.

Keywords: Herpes Zoster, Incidence, Temporal, Australia, Japan, United States

\section{Background}

Herpes Zoster (HZ) which is a reactivation of the latent Varicella Zoster Virus (VZV) [1] is primarily a disease in older adults and those with immunosuppressed conditions or treatments [2]. A population based study conducted by Yawn et al. using data between 1996 and 2005 in Olmsted County in the United States (US) reported that the risk of $\mathrm{HZ}$ increases with age; from 4.7 per 1,000 in $50-59$ year-old to 12.0 per 1,000 in $\geq 80$ year-old [3]. The study also reported that $\mathrm{HZ}$ complications, primarily Post-herpetic Neuralgia (PHN, defined as pain lasting more than 90 days from the onset

\footnotetext{
*Correspondence: lijoy.x.varghese@gsk.com

${ }^{1}$ GSK R\&D - Asia-Pacific \& North Asia, 150 Beach Road, \#22-00 Gateway West,

Singapore 189720, Singapore

Full list of author information is available at the end of the article
}

of the $\mathrm{HZ}$ rash), also increase with age; from $5.41 \%$ in $50-59$ year-old to $20.32 \%$ in $\geq 80$ year-old [3]. An extrapolation of the $\mathrm{HZ}$ incidence rate obtained from the study to the 2005 US population, results in approximately 1 million new cases of $\mathrm{HZ}$ each year [3].

Various studies [4-11] have shown that the HZ incidence increased in many countries, e.g. Australia, Canada, Japan and the US. Hales et al. [11] estimated a 39\% increase in $\mathrm{HZ}$ incidence in the $\geq 65$ year-old US Medicare insured population between 1991 and 2010. Many reasons have been cited for the increasing trend: (1) introduction of routine varicella vaccination that reduces the exposure to varicella and, thus, exogenous boosting $[12,13],(2)$ increasing aging population and the associated prevalent chronic diseases, (3) increasing prevalence of immunosuppression 
in the elderly population, and (4) immune suppression brought on by long-term stress and depression [14].

Simultaneously, the global trend of aging populations presents a challenge to healthcare resources especially with respect to diseases that impact the elderly such as HZ. The National Institute on Aging estimates that by 2030 , the world is likely to have over 1 billion people aged $\geq 65$. The oldest-old, i.e. the population aged $\geq 85$, is projected to grow by $46 \%$ between 2015 and 2030 compared to of $28 \%$ for those aged $\geq 65$ [15]. The increasing HZ incidence coupled with the demographic trends (i.e., aging population and greater life expectancy) in many countries would imply a greater economic burden of HZ on already constrained healthcare budgets.

The objective of this study is to estimate the potential burden of $\mathrm{HZ}$ (in terms of incident $\mathrm{HZ}$ cases) over time by taking into consideration the trends of $\mathrm{HZ}$ incidence and global aging. In this paper we focus on three countries for which we project the incident cases of $\mathrm{HZ}$ up to 2030 in the $\geq 65$ age cohort; the US, Japan and Australia.

\section{Methods}

A recent systematic review by Kawai et al. on the global $\mathrm{HZ}$ incidence identified 25 studies from 7 countries up to December 2013 that presented the temporal trends of HZ incidence [16]. We performed a search on PubMed to identify more recent studies using a similar search strategy [16]. We used Medical Subject Headings (MeSH) terms for 'herpes zoster' in combination with the terms 'incidence' and 'epidemiology' from January 2014 to November 2016. Studies that evaluated incidence of HZ in children or adolescents and those that assessed the rates of hospitalization arising from $\mathrm{HZ}$ were excluded. Five new studies that reported temporal trends were identified [17-21].

Data from the included studies were extracted to estimate the annual HZ incidence (per 1,000 persons) in the $\geq 65$ age cohort. If yearly incidence data was reported for different age cohorts, an aggregate age-adjusted incidence value for the $\geq 65$ age cohort was estimated using individual country demographic data obtained from the respective national statistics databases. If the incidence data was further reported based on gender, the agespecific proportion of males to females obtained from the corresponding national population databases was used to arrive at an aggregate value.

To evaluate the future burden of $\mathrm{HZ}$ the incidence rates for the various countries were projected up to the year 2030 using the following two scenarios:

(a) Scenario 1 assumes that the linear trend modeled for the corresponding country-specific annual HZ incidence data is followed till the year 2030. A linear curve was found to be the best-fit for the reported yearly incidence reported in a majority of the datasets and consequently used for the studies considered.

(b)Scenario 2 assumes a last observation carried forward value, whereby the predicted incidence based on the best fit linear curve of the last reported year of each study is carried forward (i.e. assuming a constant incidence value from the year of the conclusion of the study till the year 2030). This conservative scenario assumes that the incidence of $\mathrm{HZ}$ reaches a maximum in the last year of the study and the increase in $\mathrm{HZ}$ cases is solely an effect of the demographic change in the country

To estimate the expected number of cases up to year 2030 for both scenarios, the corresponding predicted value of incidence for each year was multiplied by the projected population estimate for same year. Population data and projections for the $\geq 65$ year old age cohort up to 2030 were obtained from United Nations World Population Prospects: the 2015 revision [16]. The medium fertility variant population projection was used which takes into account a medium fertility, normal mortality and normal international migration rates.

Although annual incidence rates were presented for 11 studies $[4-7,9,11,17,19-22]$, we focused on three studies as they reported the annual incidence data specifically for the $\geq 65$ age group while also reporting incidence over a sufficiently long time period so as to perform linear best fit curve. The studies used to estimate the incidence rates were Leung et al. [6], Toyama et al. [7] and MacIntyre et al. [4] which reported HZ incidence rates for the US, Japan and Australia respectively and was adjudged to present a reasonable picture of the expected HZ burden in high-income country setting. For the US, both Leung et al. [6] and Hales et al. [11] evaluated the incidence rates for the $\geq 65$ immunocompetent and overall population, but for our analysis we conservatively considered the former (note the incidence was consistently lower than rates reported in Hales et al.) as the yearly incidence data was readily available from the study Leung et al. [6] estimated the HZ incidence using a retrospective cohort study of medical claims data from the MarketScan (Truven Health Analytics) databases for 1993-2006. The MarketScan database is convenience sample that includes patientlevel information from over 100 self-insured employers, state governments, hospitals, health insurance plans and Medicare from all states in the United States of America. $\mathrm{HZ}$ incidence (stratified by age and sex) was calculated using all persons with a first outpatient service associated with a 053.xx code (HZ International Classification of Diseases: ICD-9 code) as the numerator, and total MarketScan enrollment as the denominator. We did not 


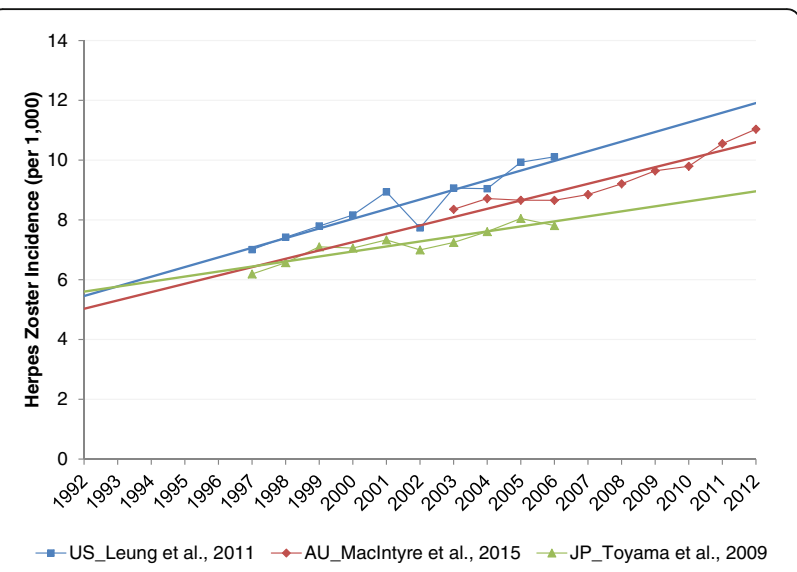

Fig. $1 \mathrm{HZ}$ incidence and trends in the Australia (AU), Japan (JP) and the United States (US)

include incidence data reported by Leung et al. [6] from 1993-1996 in our analysis as the MarketScan data during this period did not include Medicare data which represents the bulk of the US population aged 65 and above.

Toyama et al. [7] estimated the HZ incidence from the number of subjects that presented a new case of $\mathrm{HZ}$ who subsequently visited one of the 39 dermatology clinics and the dermatology departments of seven general hospitals belonging to the Miyazaki Dermatologic Society over a period of 10 years (1997-2006). Toyama et al. state that patients with $\mathrm{HZ}$ in Japan primarily consult dermatology clinics rather than practitioners of family medicine and, thus, the surveillance system covered nearly all the patients with $\mathrm{HZ}$ in the prefecture. $\mathrm{HZ}$ incidence was presented for the overall population segregated by age and gender.

MacIntyre et al. [4] presented the age specific HZ incidence based on the number of prescriptions of antivirals specific for the treatment of HZ. HZ incidence in the overall population was estimated from the number of antiviral prescriptions for $\mathrm{HZ}$ by adjusting the age specific numbers of prescriptions with the estimated proportion of new zoster cases that were prescribed direct-acting antivirals during the corresponding periods, derived from analyses of the Bettering the Evaluation and Care of Health $(\mathrm{BEACH})$ database. The BEACH database is cross-sectional paper-based data collection of a nationally representative sample of general practitioners in Australia [4].
Yawn et al. estimated the HZ-attributable healthcare costs (in 2006 US dollars) for all patients with incident cases of $\mathrm{HZ}$ among residents of Olmsted County, Minnesota in the US [23]. To estimate the projected healthcare economic burden arising from $\mathrm{HZ}$ in those aged 65 and above for the US in 2030, we used the corresponding population estimates for the US and the projected $\mathrm{HZ}$ incidence obtained using scenario 1 (mentioned earlier). The 2006 US dollar healthcare costs were adjusted for the mid-year 2030 values by extrapolating the monthly US consumer price index for medical care trends from 2005-2015 using a linear best-fit [24]. It is assumed that the proportion of incident $\mathrm{HZ}$ cases that go on to develop sequelae (PHN and other non-pain complications) [3] remain constant over the time period of analysis.

All analyses were performed using Microsoft Excel.

\section{Results}

\section{HZ Incidence}

The incidence data from the three countries showed an average annual increase of between 2.35\% [7] (Japan; Toyama et al.) and 3.74\% [6] (US; Leung et al.) over the corresponding study time periods (Fig. 1).

The incidence data for the three selected studies are shown below in Table 1.

\section{Demographics}

The population projections for the $\geq 65$ age cohort obtained from the United Nations data source are shown in Table 2. The elderly population for the US, Japan and Australia are expected to increase by 55, 10 and $53 \%$ respectively by the year 2030 compared to the levels in 2015. In 2001, $12.3 \%$ of the entire US population were aged $\geq 65$; in 2030, that proportion is estimated to rise to $20.7 \%$; a $68 \%$ increase over a period of 30 years. A similar trend is observed for Australia (Table 2). Japan, however, shows a much higher proportion of older adults (17.7\% aged 65 and above in 2001) and the proportion is estimated to increase by $71.8 \%$ in 2030 to reach $30.4 \%$ of the total population. The proportion of the population aged $\geq 80$ is expected to increase by 223 to $12.7 \%$ of the entire Japanese population in 2030.

\section{Estimated incident $\mathrm{HZ}$ cases}

In the first scenario, the number of incident $\mathrm{HZ}$ cases in the population aged $\geq 65$ in the US is expected to

Table $1 \mathrm{HZ}$ incidence (per 1,000 persons) in the Australia, Japan and the United States

\begin{tabular}{|c|c|c|c|c|c|c|c|c|c|c|c|c|c|c|c|c|}
\hline & 1997 & 1998 & 1999 & 2000 & 2001 & 2002 & 2003 & 2004 & 2005 & 2006 & 2007 & 2008 & 2009 & 2010 & 2011 & 2012 \\
\hline Leung, 2011 (United States) [6] & 7.01 & 7.42 & 7.80 & 8.16 & 8.94 & 7.73 & 9.06 & 9.04 & 9.93 & 10.11 & & & & & & \\
\hline MacIntyre, 2015 (Australia) [4] & & & & & & & 8.36 & 8.72 & 8.66 & 8.66 & 8.85 & 9.21 & 9.64 & 9.80 & 10.55 & 11.04 \\
\hline Toyama, 2009 (Japan) [7] & 6.19 & 6.57 & 7.10 & 7.06 & 7.33 & 7.00 & 7.25 & 7.61 & 8.05 & 7.81 & & & & & & \\
\hline
\end{tabular}


Table 2 Population estimates for Australia, Japan and the United States

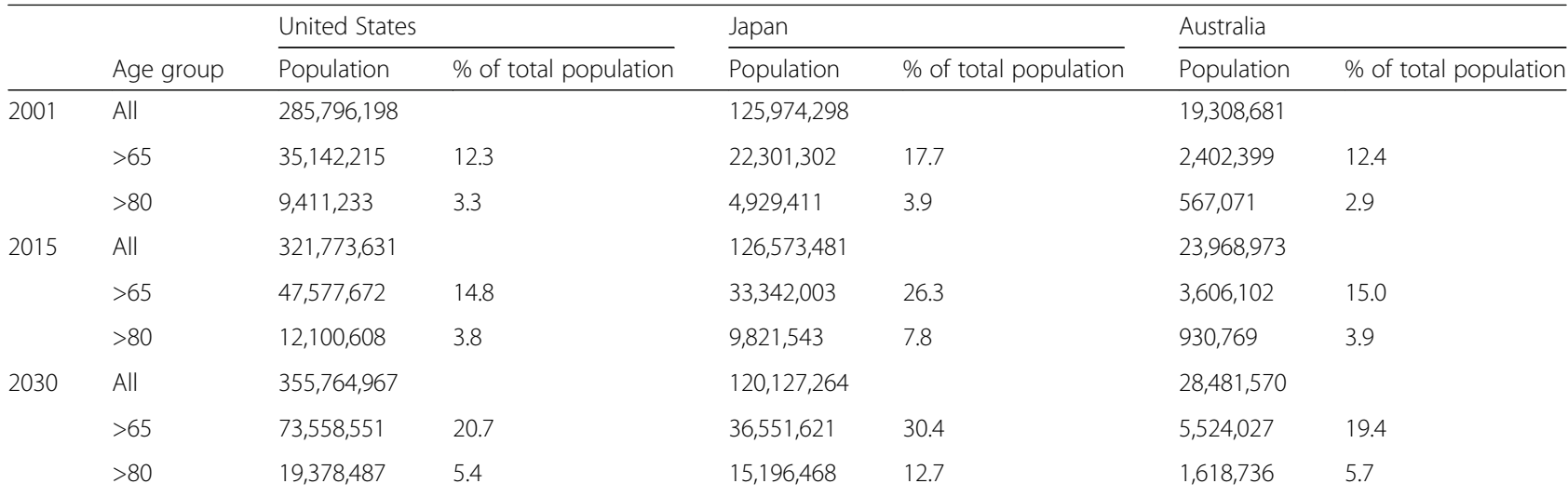

increase by $+343 \%$ (from 293,785 in 2001 to 1,303,328 in 2030) while the related increases in Japan and Australia are $+176 \%(158,616$ to 437,867$)$ and $+376 \%(18,105$ to 86,268 ) respectively (Table 3 ).

In second scenario, the percentage increases from 2001 to 2030 for the US, Japan and Australia are $+150 \%$ (293,785 to 733,629$),+83 \%(158,616$ to 290,641$)$ and $+223 \%(18,105$ to 58,561$)$ respectively (Table 3 and Figs. 2, 3 and 4).

\section{Estimated healthcare burden}

$\mathrm{HZ}$ represents a significant burden each year to the healthcare system [23]. Yawn et al. estimated the annual HZ-related costs for US population to be around 1.1 Billion (B) US\$. By adjusting the 2006 US\$ values presented in the study to the projected 2030 US\$ values (by extrapolating the published medical care cost index from January 2005 to December 2014 up to June 2030) [24], and combining it with the projected US population and $\mathrm{HZ}$ incidence for 2030, we estimated annual cost of HZrelated cases (assuming no vaccination) in the $\geq 65$ age cohort to be approximately 4.74 Billion (B) US\$ (Table 4).

The complications of $\mathrm{HZ}$ (including $\mathrm{PHN}$ and other non-pain complications), which manifest in nearly 20$25 \%$ of $\mathrm{HZ}$ cases, cost nearly three times as much as an uncomplicated case of $\mathrm{HZ}$ [23]. Of the estimated 4.74B US\$ HZ-associated costs in the US in 2030 in the $\geq 65$ years old, $62 \%$ of the costs (2.94B US\$) arise from the treatment of PHN and other complications (Table 4). Since the risk of developing HZ-associated complications (PHN and other non-pain complications) increases with age [3], the trend of the increasing proportion of the $\geq 80$ population, especially in countries like Japan, is expected to further increase the economic burden of $\mathrm{HZ}$.

\section{Discussion}

Considering the simultaneous projected increase in the elderly population and the increase in $\mathrm{HZ}$ incidence, countries like the US, Japan and Australia are expected to see an increase of 110 and $213 \%$ in $\mathrm{HZ}$ cases between 2015 and 2030 in the $\geq 65$ years old. The increase in both $\mathrm{HZ}$ incidence and the aging population is expected to be a global trend and thus the economic burden of $\mathrm{HZ}$ on healthcare systems is expected to be a global phenomenon as well.

There are limitations to the methodological approach undertaken in this study. This study used two scenarios to model and to project the temporal trend of $\mathrm{HZ}$ incidence in three chosen countries: a linear best-fit extrapolation of the reported annual incidence and a last observation carried forward method. A more rigorous approach would be to employ a time-series analysis and present the $95 \%$ confidence interval estimates for the predicted incidence in 2030. A Holt-Winters double

Table 3 Projected annual incident cases of HZ in Australia, Japan and the United States in 2030

\begin{tabular}{|c|c|c|c|c|c|c|c|c|c|c|}
\hline & & \multicolumn{3}{|c|}{ United States } & \multicolumn{3}{|l|}{ Japan } & \multicolumn{3}{|c|}{ Australia } \\
\hline & & 2001 & 2015 & 2030 & 2001 & 2015 & 2030 & 2001 & 2015 & 2030 \\
\hline \multirow[t]{2}{*}{ Scenario 1} & Projected Incidence (per 1,000) & 8.36 & 12.88 & 17.72 & 7.11 & 9.46 & 11.98 & 7.54 & 11.44 & 15.62 \\
\hline & $\mathrm{HZ}$ cases in $\geq 65$ years old & 293,785 & 612,692 & $1,303,328$ & 158,616 & 315,482 & 437,867 & 18,105 & 41,243 & 86,268 \\
\hline \multirow[t]{2}{*}{ Scenario 2} & Projected Incidence (per 1,000) & 8.36 & 9.97 & 9.97 & 7.11 & 7.95 & 7.95 & 7.54 & 10.60 & 10.60 \\
\hline & $\mathrm{HZ}$ cases $\geq 65$ years old & 293,785 & 474,511 & 733,629 & 158,616 & 265,120 & 290,641 & 18,105 & 38,229 & 58,561 \\
\hline
\end{tabular}

Scenario 1: linear extrapolation of $\mathrm{HZ}$ incidence trends observed during study period. Scenario 2: Last observation of incidence observed in the study period carried forward) HZ: Herpes Zoster 


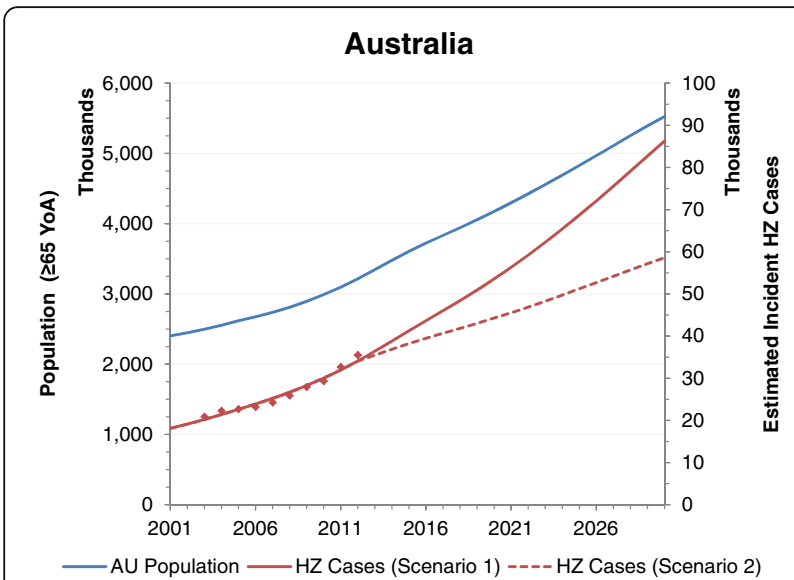

Fig. 2 Population and $\mathrm{HZ}$ incident cases estimates up to 2030 for Australia (YoA: Years of Age, HZ: Herpes Zoster; AU: Australia)

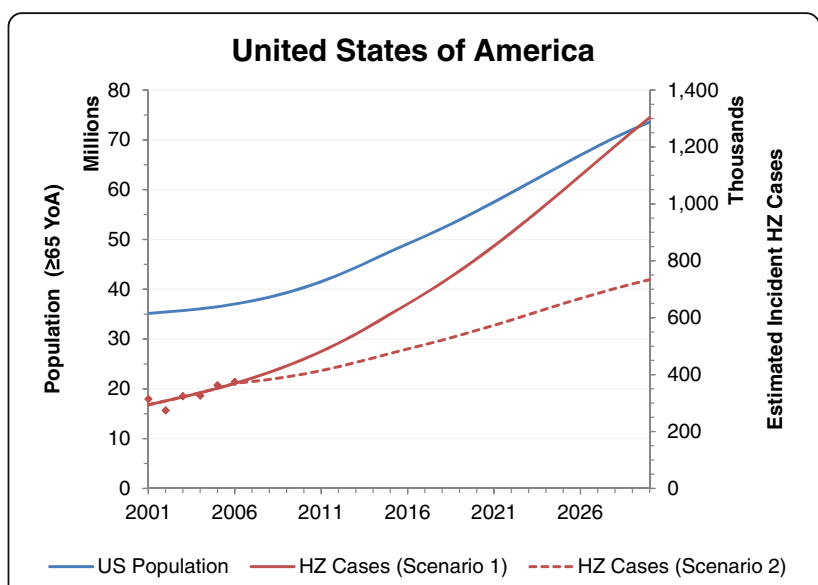

Fig. 4 Population and $\mathrm{HZ}$ incident cases estimates up to 2030 for the United States (YoA: Years of Age, HZ: Herpes Zoster; JP: Japan)

incidence will also have to be studied to model trends. Various hypotheses have been postulated, but no consensus has been reached.

One of the reasons cited for this increase is the introduction of the varicella vaccination; [13] repeated exposure of the population to naturally circulating is thought to limit the occurrence of $\mathrm{HZ}$ through exogenous boosting of immunity to the varicella virus. However, both Hales et al. [11] and Russell et al. [5] have shown that the increasing trend of $\mathrm{HZ}$ incidence was evident even before the introduction of varicella vaccination in the US and Canada, respectively. The increased $\mathrm{HZ}$ incidence is also evident in countries without varicella vaccination programs or before the introduction of the vaccine $[4,7]$. An assessment of impact modelling studies from various countries predicted that mass varicella vaccinations are expected to increase $\mathrm{HZ}$ incidence for approximately 50 years post-introduction of the vaccine [25]. However, real world evidence and modelling of the impact of varicella vaccination on $\mathrm{HZ}$ incidence is conflicting and further research is necessary to obtain a robust assessment.

In addition to the introduction of the public varicella vaccination programs, various other hypothetical factors have been attributed to the increase in $\mathrm{HZ}$ incidence such as, the aging population and associated chronic conditions and the increased prevalence of immunosuppressive conditions and treatments. Several studies, from various countries, suggest that age-specific $\mathrm{HZ}$ incidence has been increasing, i.e. the increasing incidence is observed across all age-groups $[5,11]$. Also, a casecontrol study performed in the US [26] found that while the risk of $\mathrm{HZ}$ does increase with increasing number of chronic conditions, they do not 'substantially explain' why certain people go on to develop HZ.

Immunosenescence brought on by aging has been demonstrated to be associated with the reactivation of

Fig. 3 Population and $\mathrm{HZ}$ incident cases estimates up to 2030 for Japan (YoA: Years of Age, HZ: Herpes Zoster; JP: Japan) 
Table 4 Estimated HZ-related healthcare cost burden for the $\geq 65$ in the United States in 2030

\begin{tabular}{|c|c|c|c|c|c|c|}
\hline & \multicolumn{3}{|l|}{2005} & \multicolumn{3}{|l|}{2030} \\
\hline & Cost/Case & Cases ('000 s) & $\begin{array}{l}\text { Total } \\
\text { HZ Costs (US\$) }\end{array}$ & Cost/Case & Cases ('000 s) & $\begin{array}{l}\text { Total } \\
\text { HZ Costs (US\$) }\end{array}$ \\
\hline All HZ & $\$ 1,851$ & 339 & $\$ 627,773,213$ & $\$ 3,640$ & 1,303 & $\$ 4,743,559,326$ \\
\hline $\mathrm{HZ}$ with PHN or Complications & $\$ 4,869$ & 80 & $\$ 389,153,306$ & $\$ 9,601$ & 306 & $\$ 2,937,397,732$ \\
\hline
\end{tabular}

HZ Herpes Zoster, PHN Post-herpetic Neuralgia, USI United States dollar

the latent VZV virus and thus the development of $\mathrm{HZ}$ [14]. However, Toyama et al. [7] found that the increase in $\mathrm{HZ}$ incidence was higher than the increase in the percentage of the older population. Increases in $\mathrm{HZ}$ incidence have also been observed across all age groups. It has been demonstrated that the risk of $\mathrm{HZ}$ is higher in subjects with immunosuppressive conditions or treatments. However, Leung et al. [6] found that the increase in $\mathrm{HZ}$ incidence was present in the 'healthy' population which excluded subjects that had any of the 200 identified immunosuppressive conditions or treatments.

The increasing trend of $\mathrm{HZ}$ incidence may also be due to changes in other risk factors previously identified for $\mathrm{HZ}$, e.g. increasing physical limitation or psychological stress [14, 27]. Increases could also be attributed to better diagnosis through improved access to healthcare and public awareness. This increasing trend along with the concurrent aging of the population is expected to further strain healthcare budgets. The aging population coupled with low birth rates in many countries will also result in a decline in the proportion of the working-age population [16]. The ratios of the working working-age population (15-64 years old) to the older age group (65 and above) in the US in 2001 was 5.4 and is estimated to fall to 3.9 in 2020. A similar trend is observed for Australia and the corresponding ratios in Japan are 3.8 in 2001 to 2.1 in 2020 .

A live attenuated Oka VZV vaccine which is licensed in various countries was shown to reduce $\mathrm{HZ}$ incidence by $51.1 \%$ ( $95 \%$ confidence interval, 44.2-57.6) [28]. However, vaccine efficacy was shown to reduce with age with the efficacy against $\mathrm{HZ}$ incidence dropping to $37.6 \%$ in those aged $\geq 70$ [28]. Recent results of a phase III trial of an adjuvanted HZ subunit vaccine have demonstrated a substantial improvement in vaccine efficacy in the elderly with an efficacy of 97.2 (95\% confidence interval, 93.7-99.0) in subjects $\geq 50$ years of age [29] and 91.3\% (95\% confidence interval, 86.8-94.5) in subjects $\geq 70$ years of age [30]. The two vaccines could help reduce the future global burden of $\mathrm{HZ}$ on the elderly.

\section{Conclusion}

Due to the aging of the global population, a dramatic increase in the number of people suffering from $\mathrm{HZ}$ is expected over the next few decades unless more effective preventative action is taken.

\section{Abbreviations \\ HZ: Herpes Zoster; VZV: Varicella Zoster Virus; PHN: Post-herpetic Neuralgia; MeSH: Medical Subject Headings; BEACH: Bettering the Evaluation and Care of Health; US: United States; US\$: United States dollar}

\section{Acknowledgements}

Authors would like to thank Emad Yanni for his review. Authors also thank Business \& Decision Life Sciences platform for editorial assistance and manuscript coordination, on behalf of GSK. Pierre-Paul Prevot provided editing support and Gregory Collet who coordinated manuscript development and editorial support.

Funding

GlaxoSmithKline Biologicals SA was the funding source and was involved in all study activities (GSK study identifier HO-15-16293) and overall data management (collection, analysis and interpretation). GlaxoSmithKline Biologicals SA also funded all costs associated with the development and the publishing of the present manuscript.

\section{Availability of data and materials}

All data used in this study are presented in the manuscript, or references to the original material are provided. Please contact the corresponding author should you require any additional information.

\section{Authors' contributions}

$\mathrm{DC}, \mathrm{AO}, \mathrm{BS}$ and $\mathrm{LV}$ participated to the conception and design of the study. $D C, B S$ and LV participated to the acquisition of data and DC, AO, BS and LV contributed to the analysis and interpretation of data. LV drafted the article and all co-authors revised it critically for important intellectual content. All co-authors provided final approval of the version to be submitted. All authors had full access to the data and the corresponding author was responsible for submission of the publication. All authors read and approved the final manuscript.

\section{Competing interests}

BS, DC and LV are employees of the GSK group of companies. BS and DC hold stock options in the GSK group of companies as part of their employee remuneration. $\mathrm{AO}$ is a former employee of the GSK group of companies, in charge of medical affairs activities for GSK Zoster candidate vaccine. He is currently an employee of Alexion Pharmaceuticals $\mathrm{GmbH}$.

\section{Consent for publication}

Not applicable.

Ethics approval and consent to participate

Not applicable.

\section{Author details}

${ }^{1}$ GSK R\&D - Asia-Pacific \& North Asia, 150 Beach Road, \#22-00 Gateway West, Singapore 189720, Singapore. ${ }^{2}$ GSK, Wavre, Belgium. ${ }^{3}$ Current affiliation: Alexion Pharmaceuticals, Lausanne, Switzerland.

Received: 20 July 2016 Accepted: 13 January 2017

Published online: 23 January 2017

\section{References}

1. Hope-Simpson RE. The nature of herpes zoster: a long-term study and a new hypothesis. Proc R Soc Med. 1965;58:9-20. 
2. Harpaz R, Ortega-Sanchez IR, Seward JF. Prevention of herpes zoster: recommendations of the Advisory Committee on Immunization Practices (ACIP). MMWR Recomm Rep. 2008;57:1-30.

3. Yawn BP, Saddier P, Wollan PC, St Sauver JL, Kurland MJ, Sy LS. A population-based study of the incidence and complication rates of herpes zoster before zoster vaccine introduction. Mayo Clin Proc. 2007; 82:1341-9.

4. Macintyre R, Stein A, Harrison C, Britt H, Mahimbo A, Cunningham A Increasing trends of herpes zoster in Australia. PLoS One. 2015;10:e0125025.

5. Russell ML, Dover DC, Simmonds KA, Svenson LW. Shingles in Alberta: before and after publicly funded varicella vaccination. Vaccine. 2014;32: 6319-24.

6. Leung J, Harpaz R, Molinari NA, Jumaan A, Zhou F. Herpes zoster incidence among insured persons in the United States, 1993-2006: evaluation of impact of varicella vaccination. Clin Infect Dis. 2011:52:332-40.

7. Toyama N, Shiraki K. Epidemiology of herpes zoster and its relationship to varicella in Japan: a 10-year survey of 48,388 herpes zoster cases in Miyazaki prefecture. J Med Virol. 2009;81:2053-8.

8. Jumaan AO, Yu O, Jackson LA, Bohlke K, Galil K, Seward JF. Incidence of herpes zoster, before and after varicella-vaccination-associated decreases in the incidence of varicella, 1992-2002. J Infect Dis. 2005; 191:2002-7.

9. Mullooly JP, Riedlinger K, Chun C, Weinmann S, Houston H. Incidence of herpes zoster, 1997-2002. Epidemiol Infect. 2005;133:245-53.

10. Ragozzino MW, Melton III $\amalg$, Kurland LT, Chu CP, Perry HO. Populationbased study of herpes zoster and its sequelae. Medicine (Baltimore). 1982; 61:310-6.

11. Hales CM, Harpaz R, Joesoef MR, Bialek SR. Examination of links between herpes zoster incidence and childhood varicella vaccination. Ann Intern Med. 2013;159:739-45.

12. Garnett GP, Grenfell BT. The epidemiology of varicella-zoster virus infections: the influence of varicella on the prevalence of herpes zoster. Epidemiol Infect. 1992;108:513-28.

13. Edmunds WJ, Brisson $M$. The effect of vaccination on the epidemiology of varicella zoster virus. J Infect. 2002:44:211-9.

14. Thomas SL, Hall AJ. What does epidemiology tell us about risk factors for herpes zoster? Lancet Infect Dis. 2004;4:26-33.

15. National Institute on Aging. Why Population Aging Matters: A Global Perspective. 2007

16. United Nations Department of Economic and Social Affairs. World Population Prospects, the 2015 Revision. 2012.

17. Marra F, Chong M, Najafzadeh M. Increasing incidence associated with herpes zoster infection in British Columbia, Canada. BMC Infect Dis. 2016;16:589.

18. Kawai K, Yawn BP, Wollan P, Harpaz R. Increasing incidence of herpes zoster over a 60-year period from a population-based study. Clin Infect Dis. 2016; 63:221-6.

19. Sundstrom K, Weibull CE, Soderberg-Lofdal K, Bergstrom T, Sparen P, ArnheimDahlstrom L. Incidence of herpes zoster and associated events including stroke-a population-based cohort study. BMC Infect Dis. 2015:15:488.

20. Hillebrand K, Bricout H, Schulze-Rath R, Schink T, Garbe E. Incidence of herpes zoster and its complications in Germany, 2005-2009. J Infect. 2015; 70:178-86.

21. Esteban-Vasallo MD, Gil-Prieto R, Dominguez-Berjon MF, Astray-Mochales J, Gil De Miguel A. Temporal trends in incidence rates of herpes zoster among patients treated in primary care centers in Madrid (Spain), 2005-2012. J Infect. 2014;68:378-86.

22. Pierik JG, Gumbs PD, Fortanier SA, Van Steenwijk PC, Postma MJ. Epidemiological characteristics and societal burden of varicella zoster virus in the Netherlands. BMC Infect Dis. 2012;12:110.

23. Yawn BP, Itzler RF, Wollan PC, Pellissier JM, Sy LS, Saddier P. Health care utilization and cost burden of herpes zoster in a community population. Mayo Clin Proc. 2009;84:787-94.

24. Bureau of Labor Statistics. Consumer Price Index - All Urban Consumers (CUSRO000SAM). 2015

25. World Health Organization. Varicella and herpes zoster vaccines: WHO position paper, June 2014. Releve epidemiologique hebdomadaire. 2014;89: 265-287.

26. Joesoef RM, Harpaz R, Leung J, Bialek SR. Chronic medical conditions as risk factors for herpes zoster. Mayo Clin Proc. 2012;87:961-7.

27. Irwin MR, Levin MJ, Laudenslager ML, Olmstead R, Lucko A, Lang $N$, et al. Varicella zoster virus-specific immune responses to a herpes zoster vaccine in elderly recipients with major depression and the impact of antidepressant medications. Clin Infect Dis. 2013;56:1085-93.

28. Oxman MN, Levin MJ, Johnson GR, Schmader KE, Straus SE, Gelb LD, et al. A vaccine to prevent herpes zoster and postherpetic neuralgia in older adults. N Engl J Med. 2005;352:2271-84.

29. Lal H, Cunningham AL, Godeaux O, Chlibek R, Diez-Domingo J, Hwang SJ, et al. Efficacy of an adjuvanted herpes zoster subunit vaccine in older adults. N Engl J Med. 2015;372:2087-96.

30. Cunningham AL, Lal H, Kovac M, Chlibek R, Hwang SJ, Diez-Domingo J, et al. Efficacy of the herpes zoster subunit vaccine in adults 70 years of age or older. N Engl J Med. 2016:375:1019-32.

\section{Submit your next manuscript to BioMed Central and we will help you at every step:}

- We accept pre-submission inquiries

- Our selector tool helps you to find the most relevant journal

- We provide round the clock customer support

- Convenient online submission

- Thorough peer review

- Inclusion in PubMed and all major indexing services

- Maximum visibility for your research

Submit your manuscript at www.biomedcentral.com/submit 